\title{
Analyse génétique de la résistance aux infections
}

L'analyse des gènes gouvernant le niveau de résistance de l'hôte aux micro-organismes pathogènes est grandement facilitée par l'étude de différents types de lignées génétiquement définies de souris. Le but ultime de cette recherche est, sachant prédire la prédisposition à certaines infections, de pouvoir proposer des mesures préventives, vaccinales ou autres, adaptées à chaque cas.

\section{Francine Gervais Emil Skamene}

\begin{abstract}
ADRESSE
F. Gervais : professeur à l'université McGill, docteur ès sciences. E. Skamene : professeur à que de la réponse de l'hôte aux infections bactériennes en se servant, comme outil, de populations de souris génétiquement bien définies.
\end{abstract} l'université McGill, docteur en médecine. Centre d'étude de la résistance de l'hôte de l'université McGill, institut de recherche de l'hôpital général de Montréal, 1650, avenue Cedar, Montréal, Québec, Canada H3G IA4.

TIRÉS A PART

F. Gervais.

\section{Types de lignées}

La mise en évidence de déterminants génétiques dans la résistance de l'hôte n'est possible qu'en utilisant des populations homogènes comme, par exemple, les lignées consan- a présence de microorganismes pathogènes chez l'hôte déclenche chez ce dernier une réponse qui détermine le dénouement de cette infection. On explore, depuis plusieurs années déjà, l'influence que peuvent avoir les gènes sur le contrôle de cette réponse. Nos connaissances se sont considérablement approfondies grâce à l'obtention de lignées consanguines d'animaux de laboratoires (surtout les lignées de souris qui sont particulièrement bien définies), et au développement de la méthodologie en génétique des populations et en génétique moléculaire. Cette approche a mené à l'identification de gènes contrôlant le niveau de la résistance de l'hôte à l'infection par des pathogènes viraux, bactériens et protozoaires spécifiques. Ces études ont de plus aidé à comprendre les mécanismes de défense contrôlés par ces gènes. Cette revue décrira donc les stratégies employées lors de l'analyse généti- guines de souris. Ces animaux héritent d'un lot de chromosomes identique de chaque parent (animaux homozygotes) et ont un lot d'autosomes identique dans tous leurs gamètes ; leur génotype est donc prévisible. Plusieurs types de lignées de souris sont disponibles pour ce genre d'étude : les lignées consanguines ou pures, les lignées mutantes ou coisogéniques, les lignées congéniques et les lignées recombinantes (figure 1).

Lignées consanguines. Les lignées consanguines (ou pures) proviennent de croisements frère/sœur répétés pendant 20 générations successives. Ce procédé permet de diminuer graduellement le degré d'hétérozygotie entre les souris individuelles (et donc d'augmenter le degré d'homozygotie). Quoiqu'il en soit statistiquement impossible d'atteindre une homozygotie de $100 \%$, on considère que les souris de telles lignées possèdent deux allèles identiques à chacun des loci.

Lignées mutantes ou co-isogéniques. Les lignées mutantes, pour leur part, peuvent être vues comme des «expériences de la nature » à l'intérieur d'une lignée consanguine. Elles dérivent de lignées consanguines où une mutation a eu lieu en un seul site. Ces deux lignées sont génétiquement identiques, sauf au niveau du site muté. De telles mutations (ex. : nu, bg, xid) peuvent fortement affec- 


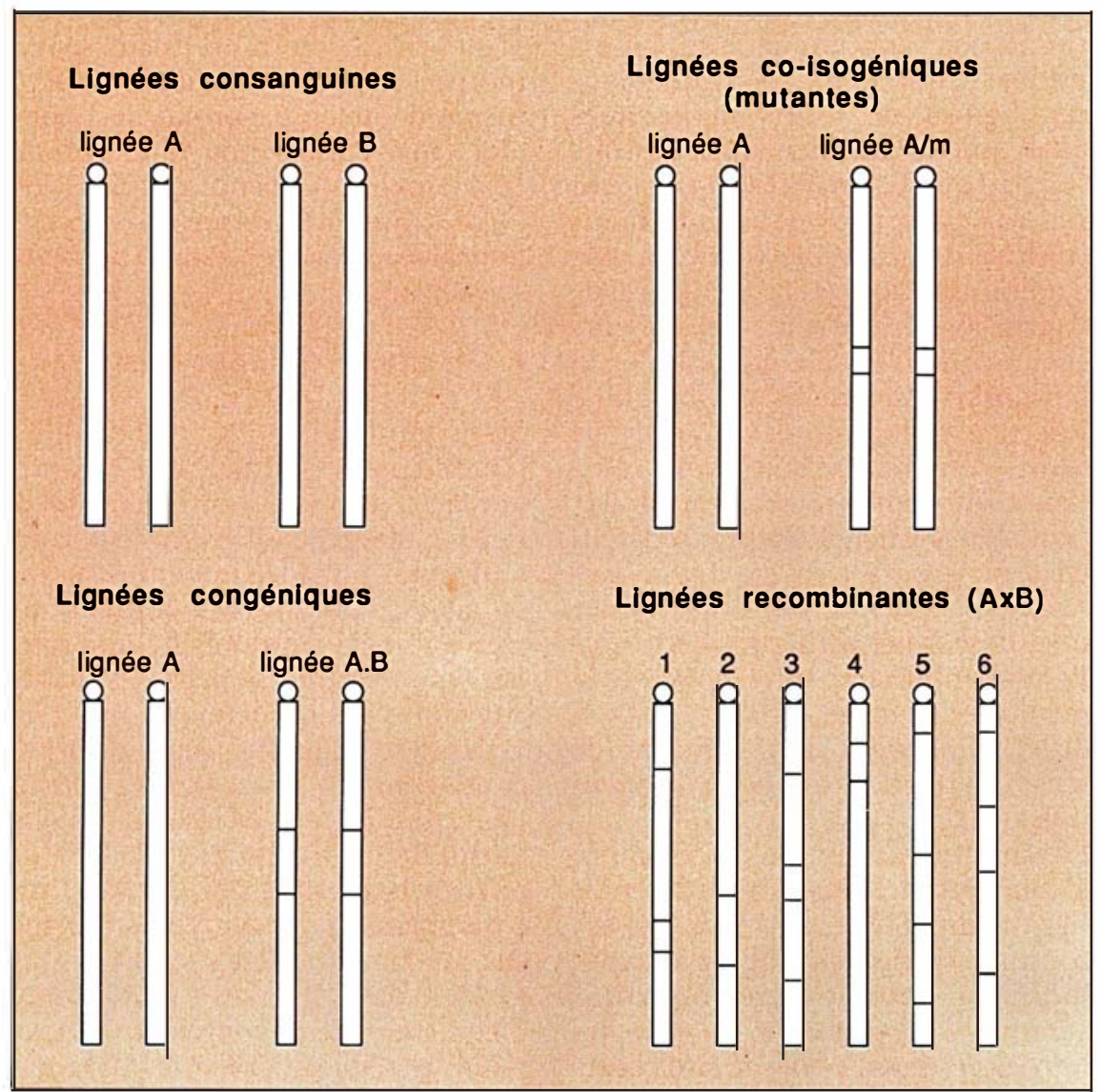

Figure 1. Différents types de lignées pures de souris utiles dans l'étude de la résistance de l'hôte à l'infection.

ter le phénotype de la résistance de l'hôte aux infections; leur étude a permis d'acquérir une meilleure compréhension des mécanismes de résistance et de l'immunité acquise [1-3].

Lignées congéniques. Les lignées congéniques sont identiques entre elles sauf pour une courte région de chromosome autour d'un marqueur choisi. Ces lignées sont généralement obtenues par le rétrocroisement (c'est-à-dire le croisement de descendants de première génération avec l'un des progéniteurs... ici celui issu d'une lignée consanguine pure, qui procurera le fond (background) génétique), pendant au moins dix générations, d'une lignée possédant un allèle connu (ex. A) (marqueur) avec une autre lignée possédant un allèle différent (ex. a) au même locus. Le but de cette manipulation est de transférer le marqueur A de la lignée donneuse à la lignée receveuse. L'éta$\mathrm{m} / \mathrm{s} n^{\circ} 9$ vol. 4 , novembre 88 lois de l'immunologie et dans l'étude des gènes de la réponse immune situés dans cette région. En revanche, un désavantage de ces lignées est dû au fait qu'une partie du chromosome entourant le gène qui sert de marqueur est inévitablement transférée de la lignée donneuse à la lignée receveuse lors de la création de la lignée congénique.

Lignées recombinantes. Les lignées consanguines recombinantes sont une addition récente importante à notre stratégie de l'analyse génétique de liaison génétique (linkage). Les lignées recombinantes dérivent de l'établissement systématique et sans sélection, de plusieurs lignées consanguines à partir de la génération F2 d'un croisement de deux lignées consanguines préexistantes et de génotype différent. Une telle série de lignées recombinantes peut être considérée comme une population ségrégante stable [4]. Chaque lignée recombinante a reçu de chacune des lignées parentales, la moitié de ses gènes autosomiques, variablement associés (figure 1). Les gènes fortement liés chez les progéniteurs auront tendance à le rester dans les lignées recombinantes.

L'utilisation d'une série de lignées recombinantes permet de vérifier si un caractère particulier est contrôlé par un seul locus, d'établir l'existence d'une liaison génétique (entre deux phénotypes), de déterminer la localisation chromosomique d'un gène et de détecter la possibilité d'effets pléiotropiques du gène.

Chaque nouveau marqueur génétique détecté dans les souches parentales peut être aisément analysé dans les lignées recombinantes où il est alors possible de déterminer s'il est où non lié à d'autres loci déjà connus et s'il consiste en une manifestation pléiotropique. Cela permet également d'apprécier si l'hérédité d'un caractère génétique est simple ou complexe, résultant par exemple de l'interaction avec des loci identifiés.

\section{Sensibilité aux infections dans des lignées consanguines de souris}

Toute analyse génétique doit débuter par l'analyse de la réponse aux infections bactériennes dans différentes 


\section{RÉFÉRENCES}

1. Roder JC. The beige mutation in the mouse. I. A stem cell predetermined impairment in natural killer cell function. J Immunol 1979; 123 : 2168-73.

2. Raff MC, Wortis HH. Thymus dependence of O-bearing cells in the peripheral lymphoid tissues of mice. Immunology 1970 ; $18: 931-42$.

3. Wicker LS, Scher I. X-linked immune deficiency (xid) of CBA/N mice. Curr Top Microbiol Immunol 1986; $124: 87-101$.

4. Taylor BA. Recombinant inbred strains of mice : use in genetic analysis of disease resistance. In: Skamene E, Kongshavn PAL Landy M, eds. Genetic Control of Natural Resistance to Infection and Malignancy. New York : Academic Press, 1980 : 1-7.

5. Wakelin D. Genetic control of susceptibility and resistance to parasitic infection. $A d v$ Parasitol 1978 ; 16 : 219-308.

6. Rosenstreich DL, WeinblattAC, O'Brien AD. Genetic control of resistance to infection in mice. Crit Rev Immunol 1982 ; 3 : 263-330.

7. Gros P, Skamene E, Forget A. Genetic control of natural resistance to Mycobacte rium bovis (BCG) in mice. J Immunol 1981; $127: 2417-21$

8. Plant J, Glynn AA. Genetics of resistance to Salmonella typhimurium in mice. J Infect Dis 1976 ; 133 : 72-8.

9. Groves MG, Osterman JV. Host defenses in experimental scrub typhus: genetics of natural resistance to infection. Infect Immun $1978 \cdot 19 \cdot 583-8$

10. Cheers C, McKenzie IFC. Resistance and susceptibility of mice to bacterial infection : genetics of listeriosis. Infect Immun 1978; 19 : lignées pures de souris. L'observation d'une variation de la réponse à un agent infectieux spécifique dans des lignées de souris maintenues dans un environnement contrôlé, suggère que la sensibilité à cet agent est un caractère génétiquement contrôlé. La dose infectieuse, la voie d'administration et la virulence de l'agent bactérien sont des facteurs critiques dans la détermination de la sensibilité. Par exemple, à une dose infectieuse donnée, les lignées de souris peuvent être soit toutes résistantes soit toutes sensibles à l'infection. En revanche, elles se séparent en deux groupes (résistantes et sensibles) lorsqu'elles sont infectées par une dose intermédiaire. Ceci souligne l'importance du choix de la méthode de typage dans les analyses de la ségrégation d'un caractère de sensibilité ou de résistance à un agent infectieux.

La variation du niveau de résistance à l'infection dans les différentes lignées peut être de deux types: continue ou discontinue [5]. L'hypothèse d'un contrôle mono (ou paucigénique) est probable lorsque les lignées de souris peuvent être clairement séparées en «sensibles » et en « résistantes». En revanche un continuum de sensibilités variables entre deux extrêmes (sensible et résistant) sera en faveur d'un contrôle polygénique du caractère, ce qui (figure 2) n'encouragera guère à entreprendre alors une étude génétique approfondie.

Dans le cas d'une distribution discontinue du caractère étudié, deux types de résultats peuvent être obtenus. Dans certains cas, les lignées peuvent ségréger d'une façon aléatoire, un nombre approximativement identique de lignées étant alors résistantes ou sensibles. Cette distribution suggère une ségrégation au hasard des allèles à un locus polymorphique (les lignées possèderont soit l'd'lèle résistant, soit l'allèle sensible). La réponse des différentes lignées de souris à certains pathogènes intracellulaires se trouve dans cette catégorie. Dans d'autres cas, une lignée ou quelques lignées seulement de souris peuvent présenter un phénotype alors que la majorité des autres lignées de souris présente l'autre phénotype. Cette distribution est généralement expliquée par une mutation [6].

L'identification d'un locus polymorphe permettra l'étude du mécanisme de défense normal de l'hôte, alors que l'apparition d'une mutation servira à identifier, dans les lignées mutantes, les mécanismes responsables de la résistance (ou de la sensibilité).

\section{Analyse de ségrégation}

Si les résultats de l'analyse de différentes lignées indiquent que le caractère de sensibilité à un agent infectieux pathogène est contrôlé par un seul gène ou par un nombre restreint de gènes, l'analyse des populations ségrégantes peut alors être amorcée. Par populations ségrégantes, nous entendons les rétrocroisements et la génération $F 2$, tous deux issus du croisement de deux lignées parentales qui diffèrent par le caractère de sensibilité au pathogène étudié. Un rétrocroisement s'obtient par un croisement d'une souris $\mathrm{Fl}$ avec une des souches parentales tandis que la génération F2 provient d'un croisement entre deux individus de première génération $(\mathrm{Fl} \times \mathrm{Fl})$. De tels croisements produisent des individus chez lesquels la distribution du caractère de sensibilité suivra les lois mendéliennes.

Cette stratégie a permis de déterminer que la résistance à l'infection par Mycobacterium bovis (BCG) est contrôlée par un seul gène autosomique dominant. L'analyse des lignées consanguines de souris démontre une ségrégation nette des différentes lignées en «résistantes » et «sensibles » au BCG (figure 3). Trois croisements entre des parents résistants et sensibles ont été réalisés. Dans tous les cas, les hybrides $\mathrm{Fl}$ de première génération se révélèrent tout autant résistants que leur parent résistant. La résistance avait donc un caractère dominant sur la sensibilité. L'analyse des individus issus des rétrocroisements et des souris de la génération F2 montra que la distribution du caractère «sensible ou résistant» était compatible avec un contrôle monogénique [7]. D’autres laboratoires ont utilisé une approche similaire pour identifier les gènes qui semblent contrôler la résistance à S. typhimurium (Ity) [8], Rickettsia t. (Ric) [9], Listeria monocytogenes 


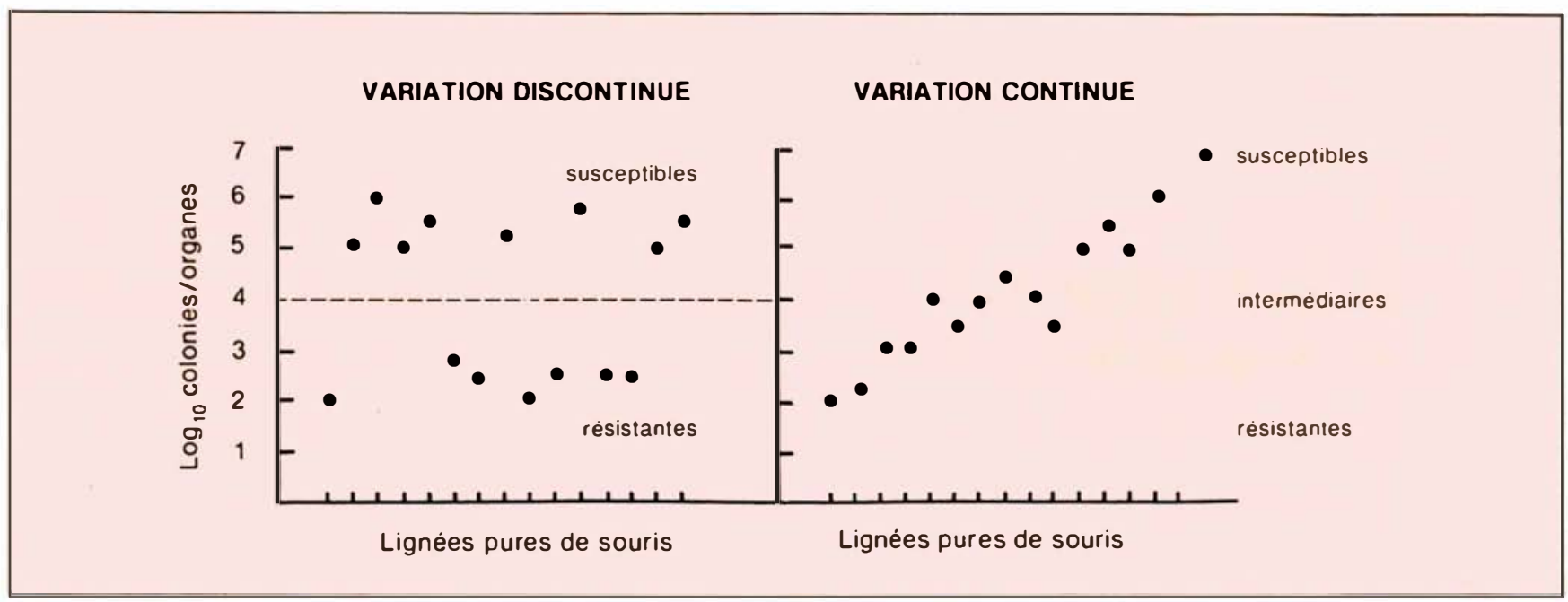

Figure 2. Contrôle génétique de la résistance de l'hôte à l'infection. Concept de l'étude du phénotype de résistance ou de sensibilité chez différentes lignées consanguines de souris. Deux variations peuvent se présenter : une variation continue ou une variation discontinue.

\begin{abstract}
Figure 3. Exemple typique d'une étude de différentes lignées consanguines de souris où le caractère montre une variation discontinue (caractère : résistance ou sensibilité au BCG). Les souris sont infectées avec une dose connue de BCG (flèche) et le nombre d'organismes BCG (nombre total d'unités formatrices de colonies par rate) est déterminé dans les rates des souris infectées, trois semaines après l'infection.
\end{abstract}

[Lr] [10], Corynebacterium kutscheri (Ack)[11], Leishmania donovani (Lsh) [12], et Influenza (Mx) [13].

Une autre approche peut apporter des informations sur le contrôle génétique de la sensibilité à une infection : les lignées de souris développées selon le modèle du Dr Biozzi [14-16]. Ces lignées ont été obtenues à la suite de croisements consécutifs où l'on sélectionnait pour le caractère phénotypique de la réponse humorale, c'est-à-dire une bonne ou mauvaise production d'anticorps. Cette stratégie provoqua l'accumulation progressive des effets des allèles gouvernant la réponse (forte ou faible) dans les lignées correspondantes. Les lignées sont ainsi devenues homozygotes à un certain nom-

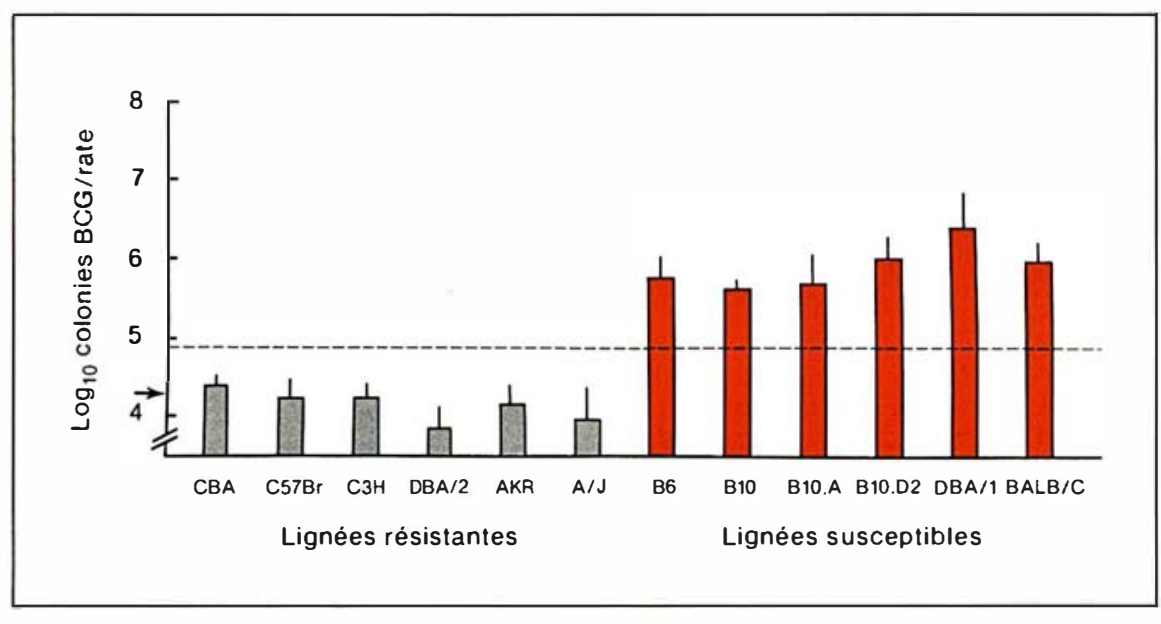

bre de loci responsables du contrôle de la réponse en anticorps (production abondante ou faible).

Lorsque ces lignées de Biozzi sont utilisées dans l'étude de la résistance aux infections, la distribution du phénotype permet de prédire le mécanisme responsable de cette résistance.

Les lignées à haute concentration d'anticorps sont les plus résistantes aux infections dont le contrôle met en jeu la réponse humorale ou une réponse cellulaire dépendante des anticorps. Au contraire, les lignées mauvaises productrices d'anticorps, résistent mieux que les autres aux agents pathogènes normalement éliminés par les macrophages. Les deux groupes de lignées de souris ont une résistance comparable aux infections influencées par une réponse inflammatoire.

\section{La carte chromosomique}

La localisation chromosomique (par rapport à d'autres marqueurs connus) du gène unique contrôlant la sensibilité à un agent infectieux est possible.

La méthode classique consiste à prendre un grand nombre d'animaux des populations ségrégantes (rétrocroisements et F2) pour les tester simultanément pour le caractère de résistance étudié et la co-expression d'une variété de marqueurs alléliques chromosomiques connus. Une autre méthode peut toutefois 


\section{RÉFÉRENCES}

11. Hirst RG, Wallace ME. Inherited resistance to Corynebacterium kutscheri in mice. Infect Immun 1982; 36 : 1257-60.

12. Bradley DJ, Taylor BA, Blackwell JM, Evans EP, Freeman J. Regulation of Leishmania within the host. III. Mapping of the locus controlling susceptibility to Leishmania in the mouse. Clin Exp Immunol 1979; $37: 7-14$.

13. Haller $O$. Inborn resistance of mice to orthomyxovirus. Curr Top Microbiol Immunol 1981 ; 92 : 25-52.

14. Biozzi G, Mouton D, Sant'Anna OA, et al. Genetics of immunoresponsiveness to natural antigens in the mouse. Curr Top Microbiol Immunol 1979 ; 85 : 31-98.

15. Biozzi G, Moulin D, Sigueira M, Stiffel C. Effect of genetic modification on murine responsiveness on anti-infection and anti-tumor resistance. In Skamene E, ed. Genetic Control of Host Resistance to Infection and Malignancy. New York: Alan Liss, 1985 : 3-18.

16. Biozzi G, Moulin D, Stiffel C, Bouthillier Y. A major role of macrophage in quantitative genetic regulation of immunoresponsiveness and anti-infectious immunity. $A d v$ Immunol $1984 ; 36$ : 189-233.

17. Plant JE, Blackwell JH, O'Brien AD, Bradley D J, Glynn AA. Are the Lsh and Ity disease resistance genes at one locus on mouse chromosome 1 ? Nature $1982 ; 277$ : 510 -1.

18. Skamene E, Gros P, Forget A, Kongshavn PAL, St. Charles C, Taylor BA. Genetic control of resistance to intracellular pathogens. Nature 1982 ; 297 : 506-9.

19. Skamene E, Kongshavn PAL, Sachs DH. Resistance to Listeria monocytogenes in mice is genetically-controlled by genes which are not linked to the H-2 complex. J Infect Dis 1979 ; 139 : 228-31.

20. Emmerling $P$, Finger $H$, Bockemuhl $J$. Listeria monocytogenes infection in nude mice. Infect Immun 1975 ; 12 : 437-9.

21. Takeya K, Shimotori S, Taniguchi T, Nomoto K. Cellular mechanisms in the protection against infection by Listeria monocytogenes in mice. J Gen Microbiol 1977 ; 100 : 373-9.

22. Newborg MF, North RJ. On the mechanism of $T$-cell-independent anti-Listeria resistance in nude mice. J Immunol 1980 ; 124: $571-6$. être utilisée: la caractérisation des lignées recombinantes pour leur sensibilité au pathogène. Un ensemble de lignées recombinantes peut être perçu comme autant de groupes d'individus identiques, ségrégeant pour un certain nombre de caractères. Ceci représente un avantage énorme comparé au besoin d'entreprendre des mesures sur des animaux isolés obtenus par rétrocroisement ou par croisement des individus [4]. Ces lignées recombinantes sont évidemment particulièrement utiles dans l'analyse génétique de caractères qui demandent que l'animal soit sacrifié pour mesurer le caractère. Une telle approche a permis de localiser le gène $B c g$ sur le chromosome 1 de la souris. Ce gène serait lié ou identique aux gènes Ity et $L s h$, qui contrôlent la réponse de l'hôte, respectivement, à la salmonellose murine et à la leishmaniose viscérale. On ne sait cependant toujours pas s'il s'agit d'un seul gène à effets pléiotropiques ou de plusieurs gènes étroitement liés [17]. Il semble, en toute éventualité, que cette région chromosomique joue sûrement un très grand rôle dans la résistance de l'hôte aux pathogènes intracellulaires en général [18], contrôlant la multiplication précoce du pathogène in vivo par l'intermédiaire de l'activité antibactérienne des macrophages tissulaires.

\section{Identification du phénotype}

L'analyse de la liaison génétique entre le caractère de résistance et un mécanisme supposé responsable de ce caractère peut être entreprise sans chercher à localiser ce gène sur la carte chromosomique. Dans ce cas, seule l'identification de l'effet pléiotropique d'un gène est recherchée. Cette approche a été utilisée pour déterminer le mécanisme responsable de la résistance naturelle à l'infection à Listeria monocytogenes. L'étude des différentes lignées consanguines a démontré une distribution du caractère de résistance en deux groupes distincts suggérant un contrôle par un gène unique dénommé $\operatorname{Lr}[10,19]$. Les mécanismes responsables de la résistance à Listeria ont été analysés en suivant le cours de l'infection chez des souris de différentes lignées consanguines.
Les cellules $T$ ne semblent pas impliquées dans la phase précoce de l'infection puisque les souris athymiques nude infectées avec Listeria ont une courbe de croissance bactérienne semblable à celle des souris normales [20-22]. Le système phagocytaire mononucléé semble en revanche conférer un degré de protection important durant cette phase précoce. En effet, l'administration d'agents connus pour inactiver le système macrophagique (comme le DS 500, la silice, les particules de carbone, la caragénine, les rayons X) aux souris infectées provoque une nette augmentation de la croissance bactérienne chez les animaux résistants [21, 23-25]. L'administration d'agents stimulant le système macrophagique (Corynebacterium parvum, BCG) aux animaux provoque au contraire une réduction de la croissance bactérienne [25, 26].

Deux types de cellules du système des phagocytes mononucléés semblent impliqués dans la résistance naturelle à Listeria: les macrophages fixés, résistant aux rayons $\mathrm{X}$ et les macrophages inflammatoires, sensibles aux rayons X [27]. Les macrophages fixés (ou tissulaires) sont responsables de l'élimination rapide des bactéries durant les six premières heures de l'infection. L'élimination des bactéries est identique chez les souris résistantes et sensibles. La fonction des macrophages tissulaires n'influencerait donc pas cette différence de résistance. Toutefois lorsque des souris résistantes sont irradiées, elles deviennent aussi sensibles à Listeria que les souris de lignées sensibles. La résistance naturelle accrue à Listeria semble donc due à un afflux plus rapide de macrophages inflammatoires capables de contrôler la multiplication des bactéries aux sites d'infection. Le phénotype «sensible» serait donc expliqué par une accumulation insuffisante de macrophages inflammatoires aux sites d'infection [24, 28, 29]. La distribution du caractère de résistance à Listeria est corrélée à celle obtenue pour la présence du facteur $\mathrm{C} 5$ (gène $H c$ ) du complément. Le facteur C5 agit comme un puissant facteur chimiotactique pour les monocytes et les macrophages lorsqu'il est activé en C5a. Le facteur C5a est normalement libéré au niveau des sites de 


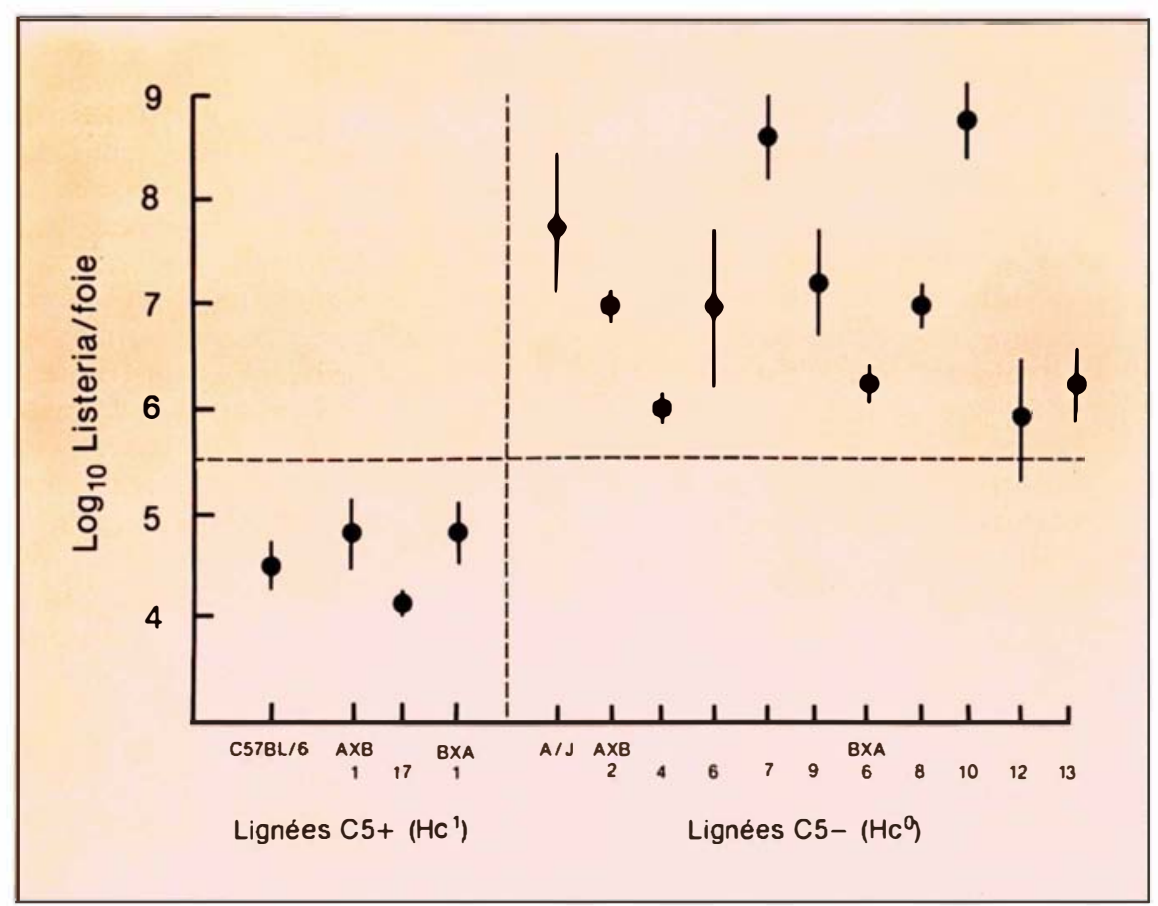

Figure 4. Distribution du caractère de résistance ou de sensibilité à Listeria dans les lignées recombinantes par rapport à la présence ou à I'absence du composé C5 du complément chez ces lignées. La résistance ou susceptibilité à Listeria est déterminée par le nombre de colonies de Listeria obtenu à partir des foies des souris infectées 72 heures auparavant (dose infectieuse : $2,5 \times 10^{4}$ Listeria).

multiplication bactérienne lorsque le facteur C5 est exposé aux composés glycolipidiques de la membrane bactérienne. Une déficience en facteur C5 dans les lignées sensibles serait donc responsable de l'absence d'un stimulus inflammatoire puissant et constituerait le mécanisme de l'expression du phénotype «sensible ».

Les deux exemples ici cités, notamment, le contrôle génétique de la résistance à Listeria et à $\mathrm{BCG}$ nous montrent l'utilité de l'analyse génétique dans l'étude des défenses de l'hôte. Cette approche a permis de déterminer que ces gènes agissent pendant la phase de résistance préimmune ou naturelle et qu'ils contrôlent un aspect précis de la réponse macrophagique à l'infection. Malgré l'importance incontestée de ces mécanismes dans la lutte précoce de l'hôte contre la multiplication de bactéries létales, il est clair que d'autres mécanismes, également sous un contrôle génétique, sont responsables de la guérison de l'hôte infecté.

$\mathrm{m} / \mathrm{s} n^{\circ} 9$ vol. 4, novembre 88 niveau du complexe $\mathrm{H}-2$ a permis ces études. Les souris congéniques, naturellement résistantes, demeurent résistantes quel que soit leur type de H-2 [31]. En revanche, les souris congéniques naturellement sensibles développent une infection systématique que seule la réponse immune dirigée par certains haplotypes $\mathrm{H}-2$ peut éliminer.

Les souris congéniques ont également été d'une importance cruciale dans la détermination de la base moléculaire du gène $M x^{+}$responsable du phénotype de résistance au virus Influenza. On savait que le mécanisme de résistance à l'Influenza par le gène $M x$ n'était pas relié aux cellules $\mathrm{T}, \mathrm{B}, \mathrm{NK}^{*}$ ou aux macrophages. De plus, il était improbable que l'interféron (IFN) comme tel puisse être le produit du gène $M x$ puisque les souris $\mathrm{Mx}^{-}$(sensibles) produisent une quantité significativement plus élevée d'IFN que les souris $\mathrm{Mx}^{+}$(résistantes) et que la résistance des souris $\mathrm{Mx}^{+}$n'était pas abolie par un traitement avec des anticorps anti-IFN. Le gène de résistance $\mathrm{Mx}^{+}$semblait moduler l'état antiviral induit par l'IFN ou coder pour un mécanisme anti-viral induit par l'IFN [32]. Les souris congéniques pour le gène $M x$ ont permis d'identifier le produit du gène $M x$ : une protéine nucléaire de $75 \mathrm{~K}$ induite par l'IFN qui inhibe spécifiquement la synthèse d'ARNm du virus Influenza [33].

\section{Conclusion}

Les études du contrôle génétique de la résistance de l'hôte aux maladies infectieuses ont pour objectif d'identifier les gènes responsables et d'analyser leur expression phénotypique aux niveaux cellulaire et moléculaire. L'identification de ces gènes se fait par l'analyse des variations entre les espèces, c'est-à-dire par une étude de la distribution des lignées de souris pour leur caractère de résistance ou de sensibilité à un agent infectieux donné. Cette distribution nous informe sur la nature de ce marqueur, c'est-à-dire s'il consiste en des allèles polymorphes retrouvés assez

\footnotetext{
* Cellules NK = natural killer, cytotoxiques, non immunes.
} 


\section{RÉFÉRENCES}

23. Mitsuyama M, Takeya K, Nomoto K, Shimotori S. Three phases of phagocyte contribution to resistance to Listeria monocytogenes. J Gen Microbiol 1975; 106 : 165-71.

24. Sadarangi C, SkameneE, Kongshavn PAL. Cellular basis of genetically determined enhanced resistance of certain mouse strains to listeriosis. Infect Immun 1980; 28 : $381-6$.

25. Finger $\mathrm{H}$, Heymer $\mathrm{B}$, Wirsing $\mathrm{CH}$, Emerling $\mathrm{P}$, Hof $\mathrm{H}$. Reversion of dextran sulfateinduced loss of antibacterial resistance by Bordetella pertussis. Infect Immun 1978; 19 : 950-60.

26. Matsuo K, Takeya K, Nomoto K, Shimotori S, Terasaka P. T-cell independent deviation of macrophages by viable BCG in tumorbearing mice. Cell Immunol 1981; 57 : 290306.

27. Volkman A, Collins FM. The restorative effect of peritoneal macrophages on delayed hypersensitivity following ionizing radiations. Cell Immunol 1971 ; 2 : 552-66.

28. Kongshavn PAL. Genetic control of resistance to Listeria infection. Curr Top Microbiol Immunol 1986; 124 : 67-85.

29. Stevenson MM, Kongshavn PAL, Skamene $\mathrm{E}$. Genetic linkage of resistance to $\mathrm{Lis}$ teria monocytogenes with macrophage inflammatory responses. J Immunol 1981; 127 : 402-7.

30. Gervais F, Stevenson M, Skamene E. Genetic control of resistance to Listeria monocytogenes: regulation of leukocyte inflammatory responses by the Hc locus. $J$ Immunol 1984; 132 : 2078-83.

31. Blackwell J, Freeman J, Bradley D. Influence of $\mathrm{H}-2$ complex on acquired resistance to Leishmania donovani infection in mice. Nature $1980 ; 283: 72-4$.

32. Haller $\mathrm{O}$, Arnheiter $\mathrm{H}$, Lindenmann J, Gresser I. Host gene influences sensitivity to interferon action selectivity for Influenza virus. Nature $1980 ; 283$ : 660-2

33. Krug RM, Shaw M, Broni B, Shapiro G, Haller O. Inhibition of influenza viral mRNA synthesis in cells expressing the interferon-induced Mx gene product. J Virol 1985 ; 56 : $201-6$.

34. Medina S, Vas SI, Robson HG. Effect of non-specific stimulation on the defense mechanisms of inbred mice. J Immunol $1975 ; 114: 1720-5$.

35. Perez H, Labrador F, Ledzema E. The response of therapeutical treatment of resistant and susceptible mice infected with Leishmania menicana. In: Skamene E, Kongshavn PAL, Landy M, eds. Genetic Control of Natural Resistance to Infection and Malignancy. New York: Academic Press, 1980 : 47 - fréquemment dans la population ou s'il s'agit d'une mutation rencontrée seulement dans quelques lignées.

Le mode de transmission peut ensuite être déterminé par une analyse mendélienne des populations ségrégantes (générations $\mathrm{F} 2$, rétrocroisement). Cette analyse de ségrégation est utile pour établir si un caractère particulier est sous un contrôle monogénique. Si tel est le cas, la localisation chromosomique et l'analyse de liaison génétique peuvent être entreprises afin d'identifier le gène et de déterminer sa liaison avec d'autres gènes. Ces derniers peuvent ne servir que de marqueurs, ou bien être impliqués dans l'expression phénotypique du caractère.

L'utilisation des lignées recombinantes dans ces études s'est révélée d'un immense intérêt dans l'établissement et la localisation chromosomique et dans les analyses de liaison génétique. La carte chromosomique du génome murin est de plus en plus complète, surtout depuis l'avènement des allèles RFLP (restriction fragment length polymorphism alleles) qui permettent de localiser un gène sans avoir à identifier son expression phénotypique.

Les travaux en vue de cloner le gène $B c g$-Ity-Lsh ont révélé un groupe de liaison caractérisé par des allèles RFLP encadrant ce locus. Ce groupe de liaison est conservé chez d'autres espèces (chez l'humain et les bovins). Il pourrait donc exister un gène humain équivalent à ce gène murin. Le clonage du gène Bcg-Ity-Lsh pourrait donc faciliter l'établissement de marqueurs génétiques qui permettraient d'identifier dans la population humaine les individus sensibles à la tuberculose, à la lèpre ou à d'autres types d'infections semblables.

De plus, ces efforts peuvent mener à l'élucidation du mécanisme de quelques phénotypes de résistance en les liant à des gènes codant pour certaines enzymes ou autres protéines qui peuvent jouer un rôle dans la résistance en général. Nous avons vu un tel exemple avec l'association entre le gène $\mathrm{Hc}$ codant pour la présence du facteur C5 du complément et la résistance à Listeria. Une telle approche peut aussi aboutir à l'identification de marqueurs servant à déterminer les individus sensibles ou résistants dans la population en général sans avoir à recourir à des méthodes destructrices. Chez les mammifères, de longs segments de chromosomes ont été conservés à travers l'évolution. Dans plusieurs cas, on a retrouvé des séquences homologues entres les gènes murins et humains de résistance. La connaissance de la localisation et de la structure du gène de résistance chez la souris peut donc directement mener à l'identification de son analogue humain.

L'autre aspect de l'analyse génétique de la résistance de l'hôte aux infections est la détermination de son mécanisme. Les populations ségrégantes et les lignées congéniques permettent de vérifier l'importance du mécanisme dans le contrôle de l'expression phénotypique du gène. Les lignées congéniques facilitent la tâche en réduisant au minimum le «bruit de fond » du reste du génome. Finalement, l'utilisation de ces variations dans la réponse de l'hôte obtenues avec les lignées pures de souris est d'une importance primordiale dans le développement de stratégies de vaccinations. Les modèles murins ont souvent démontré que le succès de la vaccination dépend (de façon cruciale) de la résistance naturelle à l'infection ou de la qualité de la réponse immune acquise engendrée chez l'hôte génétiquement résistant ou sensible [34, 35]. Nos connaissances de ces variations de l'efficacité d'un vaccin devraient être employées pour (a) identifier les épitopes conférant une immunoprotection chez l'hôte naturellement résistant aussi bien que sensible et (b) pour développer une stratégie appropriée pour pallier l'incapacité génétique d'élaborer une réponse adéquate au vaccin. Le récent échec du programme de vaccination contre la tuberculose endémique en Inde pourrait en partie être attribué à un tel manque d'informationssurcetaspect du problème. La raison de poursuivre l'étude de la génétique de résistance aux agents infectieux est évidente: pouvoir identifier dans la population les individus génétiquement sensibles. Ceci permettrait d'agir de façon sélective au niveau de la prévention et de la détection précoce des maladies infectieuses 


\section{Summary}

Introduction of pathogenic microorganisms into the host triggers a response which determines the outcome of infection. The possibility that genetic influences may govern such response has long been suspected and explored. A precise knowledge of this aspect, however, has become available only very recently. The use of well-defined inbred strains of laboratory animals, namely mice, and the development of methodologic advances in population and molecular genetics contributed to a clearer view of this aspect and permitted investigators to identify genes responsible for determining the level of resistance to specific viral, bacterial and protozoal pathogens. Such studies proved to be of great value to our understanding of normal defence mechanisms regulated by those genes. This review will focus on the strategies of the use of geneticallydefined populations of mice as the tool in the genetic analysis of host responses to bacterial infections.

\section{ASSOCIATION CLAUDE BERNARD}

\section{Sessions d'initiation aux modèles expérimentaux}

L'Association Claude Bernard, pour le développement des Recherches Biologiques et Médicales dans les Hôpitaux de l'Assistance Publique à Paris, organise dans ses Centres et Formations Associées en 1988-1989, des sessions de techniques expérimentales ou d'investigations. Les principaux thèmes sont la micro-chirurgie expérimentale, des techniques expérimentales cardio-vasculaires, l'étude biochimique des glomérules isolés, le métabolisme hépatique in situ et in vitro, l'étude biochimique expérimentale de l'oreille interne, les animaux âgés, l'hybridation in situ, les greffes et les cultures de tissu nerveux, les dosages radioenzymatiques et la culture de cellules neuroendocriniennes, l'analyse d'images (ganglions et moelle) et les techniques de flux en hématologie, la transplantation intestinale expérimentale, ainsi que trois enseignements de méthodologie de l'essai thérapeutique.

Une affiche générale annonçant les sessions sera adressée à tous ceux qui le demanderont.

Pour tout renseignement, écrire à :

Association Claude Bernard, Assistance Publique

3, avenue Victoria, 75100 Paris RP

Tél. : (1) 40.27.35.94
Le Président du Conseil Scientifique

Gabriel Richet 